\title{
Masking of contour-detectors in the human visual system'
}

ALBERTA S. GILINSKY

UNIVERSITY OF BRIDGEPORT

Inspection of horizontal, vertical, or diagonally oriented line gratings differentially affects the ability to detect test contours as a function of their orientation. The orientationspecific effects on visual acuity are systematic functions of the duration of adaptation to the patterns of light and dark lines.

The ability to perceive a visual display following exposure to an adapting flash is known to be impaired by increasing the brightness or the duration of the adapting flash (Brown, 1964; Brown, Graham, Leibowitz, \& Ranken, 1953; Diamond \& Gilinsky, 1955). The effect on visual acuity of exposure to adapting flashes which are patterned in various ways does not seem to have been investigated, although different patterns of adapting light might be expected selectively to influence the subsequent visual discrimination. The major purpose of the present study was to determine whether exposures of various durations to lines in one axis orientation would selectively destroy dark adaptation and raise the visual acuity threshold for lines in that specific orientation more than for lines in other orientations.

\section{Method}

This experiment investigated the probability of seeing a fine acuity grating at threshold energy value immediately following the exposure to a $1.2 \log \mathrm{ft}-\mathrm{L}$ adapting field containing the identical grating in each one of four different orientations: horizontal, vertical, $45^{\circ}$ and $135^{\circ}$. The apparatus was a Scientific Prototype two channel tachistoscope. The acuity gratings used in both the adapting field and the test field were opaque black and white lines $1 \mathrm{~mm}$ wide and required .31 acuity. The test field was centered in the fleld of the adapting display and set at a constant luminance value of $.94 \log \mathrm{ft}-\mathrm{L}$. The duration of the test flash was varied to determine that duration which would give $50 \%$ correct responses following a nonstriate adapting exposure of the same total Iuminance as the striate adapting field. These threshold settings provided a baseline against which to compare the probability of detection of the test orientation following the exposure of patterns of light and dark lines. A range of adapting durations from .1 to $8 \mathrm{sec}$ was employed.

Every experimental session began with $10 \mathrm{~min}$ of dark adapation. At the ready signal, $S$ positioned his head in the hood of the tachistoscope. Both eyes were used. There was no flxation point, and $S$ was free to
DETECTION OF HORIZONTAL LINES GK

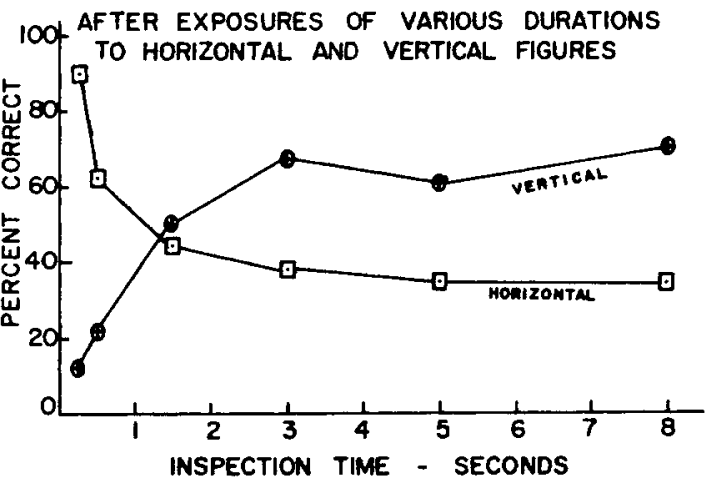

Fig. 1. Probability of detection of horizontal lines after exposures of various durations to horizontal inspection patterns (open squares) and vertical patterns (crossed circles) for observer GK.

scan the displays. His task was to report the position of the test grating which was randomized from trial to trial. Catch trials, in which no line target but a blank test flash of matched average luminance, were interspersed in $10 \%$ of the trials.

Four university students with normal or corrected vision served as Ss.

\section{Resulis}

The results are presented in the form of the probability of identification of the test grating orientation, the percent correct, under each condition. Figures 1 and 2 show the effects on detection of horizontal and vertical lines of exposures of various durations to horizontal and vertical displays. Note that the curve for the identical orientations of inspection and

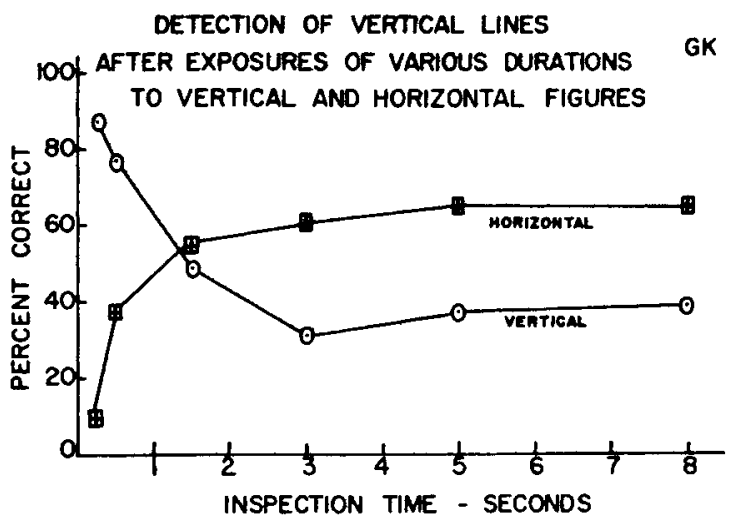

Fig. 2. Probability of detection of vertical lines after exposures of various durations to vertical inspection patterns (open circles) and horizontal patterns (crossed squares) for observer GK. 
BRIEF ADAPTING PATTERN $(250 \mathrm{~ms}$.
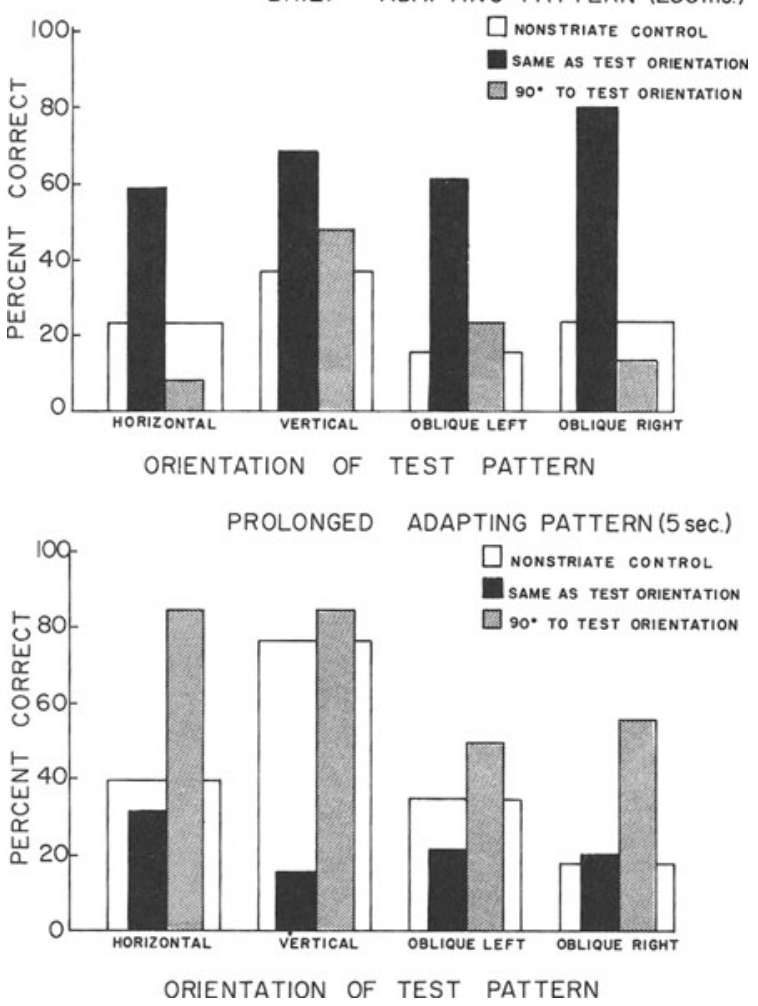

Figs. 3. \& 4. Probability of detection of test orientations following brief and prolonged exposures to adapting striate and nonstriate displays.

test stimuli (Fig. 1-both horizontal; Fig. 2-both vertical) starts at a high level and drops below the $50 \%$ point at $1.5 \mathrm{sec}$ adapting exposure. The curve for the orthogonal orientations of inspection and test gratings starts at a low probability value, climbs, and crosses the other curve at $1.5 \mathrm{sec}$. The separation between the two curves is maintained as exposure duration increases. Thus, lengthening the exposure to adapting lines appears to mask or inhibit the perception of similarly oriented lines but to facilitate the perception of lines oriented at $90^{\circ}$ to the initial stimuli. The results do not discriminate between the inhibition by identical lines and the facilitation by the orthogonal lines.

In order to separate the inhibitory from the facilitatory effects, we need to compare the results of the different combinations of test and inspection line orientations with the results of blank or nonstriate light-adapting control exposures. Figures 3 and 4 show these comparisons for a single $S$. These are representative data for brief exposures and for prolonged exposures to the adapting patterned and unpatterned stimuli. The data for all Ss agree in showing that brief exposures facilitate the identification of the same orientation, while long exposures inhibit or mask lines with the same orientation.

\section{Discussion}

The results clearly show aftereffects of achromatic patterned stimulation with fine gratings of light and dark lines. Evidently these effects are determined by the orientation of the lines in the test pattern in relation to the orientation of the lines in the inspection pattern. They resemble the orientation-specific aftereffects of color adaptation found by McCollough (1965) and the higher order receptive fleld organizations found in the cat's visual cortex (Hubel, 1963; Hubel \& Wiesel, 1962, 1965). The present experiments indicate that contour or line detectors representing cone functioning in the human visual system are orientation-specific and subject to light and dark adaptation, responding both with decreased sensitivity to lines in those spatial directions with which they have recently been prolongedly stimulated, and with increased sensitivity to lines rotated $90^{\circ}$ from the adapting patterns.

\section{References}

Brown, J. Time required for detection of acuity targets following exposure to short adapting flashes. J. Engng. Psychol., 1964, $3,53-71$.

Brown, J. L., Graham, C. H., Leibowitz, H., \& Ranken, H. Luminance thresholds for the resolution of visual detail during dark adaptation. J. Opt. Soc. Amer., 1953, 43, 197-202.

Diamond, A. L., \& Gilinsky, A. S. Dark-adaptation luminance thresholds for the resolution of detail following different durations of light adaptation. J. exp. Psychol., 1955, 50, 134-143.

Hubel, D. H. Integrative processes in central visual pathways of the cat. J. Opt. Soc. Amer., 1963, 53, 58-66.

Hubel, D. H., \& Wiesel, T. N. Receptive fields, binocular interaction, and functional architecture in the cat's visual cortex. J. Physiol., 1962, 160, 106-154.

Hubel, D. H., \& Wiesel, T. N. Receptive fields and functional architecture in two nonstriate visual areas (18 and 19) of the cat. J. Neurophysiol., 1965, 28, 229-289.

McCollough, $C$. Color adaptation of edge-detectors in the human visual system. Science, 1965, 149, 1115-1116.

\section{Notes}

1. This work was supported in part by National Science Foundation Grant GB-6067.

2. Pilot studies now in progress at the University of Bridgeport Laboratory of Psychology indicate parallel masking effects of wide stripes, mediating rod acuity functions. 